\title{
Contraction mapping principle with generalized altering distance function in ordered metric spaces and applications to ordinary differential equations
}

\section{Yongfu Su*}

"Correspondence: suyongfu@gmail.com Department of Mathematics, Tianjin Polytechnic University, Tianjin, 300387, P.R. China

\section{Springer}

\begin{abstract}
The aim of this paper is to present the definition of a generalized altering distance function and to extend the results of Yan et al. (Fixed Point Theory Appl. 2012:152, 2012) and some others, and to prove a new fixed point theorem of generalized contraction mappings in a complete metric space endowed with a partial order by using generalized altering distance functions. The results of this paper can be used to investigate a large class of nonlinear problems. As an application, we discuss the existence of a solution for a periodic boundary value problem.
\end{abstract}

Keywords: contraction mapping principle; partially ordered metric spaces; fixed point; generalized altering distance function; ordinary differential equations

\section{Introduction}

The Banach contraction mapping principle is a classical and powerful tool in nonlinear analysis. Weak contractions are generalizations of the Banach contraction mapping, which have been studied by several authors. In [1-8], the authors prove some types of weak contractions in complete metric spaces, respectively. In particular, the existence of a fixed point for weak contractions and generalized contractions was extended to partially ordered metric spaces in [2, 9-22]. Among them, some involve altering distance functions. Such functions were introduced by Khan et al. in [1], where they present some fixed point theorems with the help of such functions. First, we recall the definition of an altering distance function.

Definition 1.1 An altering distance function is a function $\psi:[0, \infty) \rightarrow[0, \infty)$ which satisfies:

(a) $\psi$ is continuous and non-decreasing.

(b) $\psi=0$ if and only if $t=0$.

Recently, Harjani and Sadarangani proved some fixed point theorems for weak contractions and generalized contractions in partially ordered metric spaces by using the altering distance function in [11, 23], respectively. Their results improve the theorems of $[2,3]$.

(C) 2014 Su; licensee Springer. This is an Open Access article distributed under the terms of the Creative Commons Attribution License (http://creativecommons.org/licenses/by/2.0), which permits unrestricted use, distribution, and reproduction in any medium, provided the original work is properly cited. 
Theorem 1.2 ([11]) Let $(X, \leq)$ be a partially ordered set and suppose that there exists a metric $d \in X$ such that $(X, d)$ is a complete metric space. Let $f: X \rightarrow X$ be a continuous and non-decreasing mapping such that

$$
d(f(x), f(y)) \leq d(x, y)-\psi(d(x, y)), \quad \text { for } x \geq y
$$

where $\psi:[0, \infty) \rightarrow[0, \infty)$ is continuous and non-decreasing function such that $\psi$ is positive in $(0, \infty), \psi(0)=0$ and $\lim _{t \rightarrow \infty} \psi(t)=\infty$. If there exists $x_{0} \in X$ with $x_{0} \leq f\left(x_{0}\right)$, then $f$ has a fixed point.

Theorem 1.3 ([23]) Let $(X, \leq)$ be a partially ordered set and suppose that there exists a metric $d \in X$ such that $(X, d)$ is a complete metric space. Let $f: X \rightarrow X$ be a continuous and non-decreasing mapping such that

$$
\psi d(f(x), f(y)) \leq \psi(d(x, y))-\phi(d(x, y)), \quad \text { for } x \geq y,
$$

where $\psi$ and $\phi$ are altering distance functions. If there exists $x_{0} \in X$ with $x_{0} \leq f\left(x_{0}\right)$, then $f$ has a fixed point.

Subsequently, Amini-Harandi and Emami proved another fixed point theorem for contraction type maps in partially ordered metric spaces in [10]. The following class of functions is used in [10].

Let $\Re$ denote the class of those functions $\beta:[0, \infty) \rightarrow[0,1)$ which satisfy the condition: $\beta\left(t_{n}\right) \rightarrow 1 \Rightarrow t_{n} \rightarrow 0$.

Theorem $1.4([10])$ Let $(X, \leq)$ be a partially ordered set and suppose that there exists a metric $d$ such that $(X, d)$ is a complete metric space. Let $f: X \rightarrow X$ be an increasing mapping such that there exists an element $x_{0} \in X$ with $x_{0} \leq f\left(x_{0}\right)$. Suppose that there exists $\beta \in \Re$ such that

$$
d(f(x), f(y)) \leq \beta(d(x, y)) d(x, y) \quad \text { for each } x, y \in X \text { with } x \geq y .
$$

Assume that either $f$ is continuous or $M$ is such that if an increasing sequence $x_{n} \rightarrow x \in X$, then $x_{n} \leq x, \forall n$. Besides, if for each $x, y \in X$ there exists $z \in m$ which is comparable to $x$ and $y$, then $f$ has a unique fixed point.

In 2012, Yan et al. proved the following result.

Theorem 1.5 ([24]) Let $X$ be a partially ordered set and suppose that there exists a metric $d$ in $x$ such that $(X, d)$ is a complete metric space. Let $T: X \rightarrow X$ be a continuous and non-decreasing mapping such that

$$
\psi(d(T x, T y)) \leq \phi(d(x, y)), \quad \forall x \geq y,
$$

where $\psi$ is an altering distance function and $\phi:[0, \infty) \rightarrow[0, \infty)$ is a continuous function with the condition $\psi(t)>\phi(t)$ for all $t>0$. If there exists $x_{0} \in X$ such that $x_{0} \leq T x_{0}$, then $T$ has a fixed point. 
The aim of this paper is to present the definition of generalized altering distance function and to extend the results of Yan et al. [24] and some others, and to prove a new fixed point theorem of generalized contraction mappings in a complete metric space endowed with a partial order by using generalized altering distance functions. The results of this paper can be used to investigate a large class of nonlinear problems. As an application, we discuss the existence of a solution for a periodic boundary value problem.

\section{Main results}

We first give the definition of generalized altering distance function as follows.

Definition 2.1 A generalized altering distance function is a function $\psi:[0, \infty) \rightarrow[0, \infty)$ which satisfies:

(a) $\psi$ is non-decreasing;

(b) $\psi=0$ if and only if $t=0$.

We first recall the following notion of a monotone non-decreasing function in a partially ordered set.

Definition 2.2 If $(X, \leq)$ is a partially ordered set and $T: X \rightarrow X$, we say that $T$ is monotone non-decreasing if $x, y \in X, x \leq y \Rightarrow T(x) \leq T(y)$.

This definition coincides with the notion of a non-decreasing function in the case where $X=R$ and $\leq$ represents the usual total order in $R$.

In what follows, we prove the following theorem, which is the generalized type of Theorems 1.2-1.5.

Theorem 2.3 Let $X$ be a partially ordered set and suppose that there exists a metric $d$ in $x$ such that $(X, d)$ is a complete metric space. Let $T: X \rightarrow X$ be a continuous and nondecreasing mapping such that

$$
\psi(d(T x, T y)) \leq \phi(d(x, y)), \quad \forall x \geq y,
$$

where $\psi$ is a generalized altering distance function and $\phi:[0, \infty) \rightarrow[0, \infty)$ is a right upper semi-continuous function with the condition: $\psi(t)>\phi(t)$ for all $t>0$. If there exists $x_{0} \in X$ such that $x_{0} \leq T x_{0}$, then $T$ has a fixed point.

Proof Since $T$ is a non-decreasing function, we obtain by induction that

$$
x_{0} \leq T x_{0} \leq T^{2} x_{0} \leq T^{3} x_{0} \leq \cdots \leq T^{n} x_{0} \leq T^{n+1} x_{0} \leq \cdots .
$$

Put $x_{n+1}=T x_{n}$. Then, for each integer $n \geq 1$, from (1) and, as the elements $x_{n+1}$ and $x_{n}$ are comparable, we get

$$
\psi\left(d\left(x_{n+1}, x_{n}\right)\right)=\psi\left(d\left(T x_{n}, T x_{n-1}\right)\right) \leq \phi\left(d\left(x_{n}, x_{n-1}\right)\right) .
$$

Using the condition of Theorem 2.3 we have

$$
d\left(x_{n+1}, x_{n}\right)<d\left(x_{n}, x_{n-1}\right) .
$$


Hence the sequence $\left\{d\left(x_{n+1}, x_{n}\right)\right\}$ is decreasing and, consequently, there exists $r \geq 0$ such that

$$
d\left(x_{n+1}, x_{n}\right) \rightarrow r^{+}
$$

as $n \rightarrow \infty$. Consider the properties of $\psi$ and $\phi$, letting $n \rightarrow \infty$ in (2) we get

$$
\psi(r) \leq \lim _{n \rightarrow \infty} \psi\left(d\left(x_{n+1}, x_{n}\right)\right) \leq \lim _{n \rightarrow \infty} \phi\left(d\left(x_{n}, x_{n-1}\right)\right) \leq \phi(r) .
$$

By using the condition: $\psi(t)>\phi(t)$ for all $t>0$, we have $r=0$, and hence

$$
d\left(x_{n+1}, x_{n}\right) \rightarrow 0,
$$

as $n \rightarrow \infty$. In what follows, we will show that $\left\{x_{n}\right\}$ is a Cauchy sequence. Suppose that $\left\{x_{n}\right\}$ is not a Cauchy sequence. Then there exists $\varepsilon>0$ for which we can find subsequences $\left\{x_{n_{k}}\right\}$ with $n_{k}>m_{k}>k$ such that

$$
d\left(x_{n_{k}}, x_{m_{k}}\right) \geq \varepsilon
$$

for all $k \geq 1$. Further, corresponding to $m_{k}$ we can choose $n_{k}$ in such a way that it is the smallest integer with $n_{k}>m_{k}$ and satisfying (5). Then

$$
d\left(x_{n_{k-1}}, x_{m_{k-1}}\right)<\varepsilon
$$

From (5) and (6), we have

$$
\varepsilon \leq d\left(x_{n_{k}}, x_{m_{k}}\right) \leq\left(d\left(x_{n_{k}}\right), x_{n_{k-1}}\right)+d\left(x_{n_{k-1}}, x_{m_{k}}\right)<d\left(x_{n_{k}}, x_{n_{k-1}}\right)+\varepsilon .
$$

Letting $k \rightarrow \infty$ and using (4), we get

$$
\lim _{k \rightarrow \infty} d\left(x_{n_{k}}, x_{m_{k}}\right)=\varepsilon
$$

By using the triangular inequality we have

$$
\begin{aligned}
& d\left(x_{n_{k}}, x_{m_{k}}\right) \leq d\left(x_{n_{k}}, x_{n_{k-1}}\right)+d\left(x_{n_{k-1}}, x_{m_{k-1}}\right)+d\left(x_{m_{k-1}}, x_{m_{k}}\right), \\
& d\left(x_{n_{k-1}}, x_{m_{k-1}}\right) \leq d\left(x_{n_{k-1}}, x_{n_{k}}\right)+d\left(x_{n_{k}}, x_{m_{k}}\right)+d\left(x_{m_{k}}, x_{m_{k-1}}\right) .
\end{aligned}
$$

Letting $k \rightarrow \infty$ in the above two inequalities and using (4) and (7), we have

$$
\lim _{k \rightarrow \infty} d\left(x_{n_{k-1}}, x_{m_{k-1}}\right)=\varepsilon
$$

As $n_{k}>m_{k}$ and $x_{n_{k-1}}$ and $x_{m_{k-1}}$ are comparable, using (1) we have

$$
\psi\left(d\left(x_{n_{k}}, x_{m_{k}}\right)\right) \leq \phi\left(d\left(x_{n_{k-1}}, x_{m_{k-1}}\right)\right) .
$$


Consider the properties of $\psi$ and $\phi$, letting $k \rightarrow \infty$ and taking into account (7) and (8), we have

$$
\psi(\varepsilon) \leq \phi(\varepsilon)
$$

From the condition $\psi(t)>\phi(t)$ for all $t>0$, we get $\varepsilon=0$, which is a contradiction. This shows that $\left\{x_{n}\right\}$ is a Cauchy sequence and, since $X$ is a complete metric space, there exists $z \in X$ such that $x_{n} \rightarrow z$ as $n \rightarrow \infty$. Moreover, the continuity of $T$ implies that

$$
z=\lim _{n \rightarrow \infty} x_{n+1}=\lim _{n \rightarrow \infty} T x_{n}=T z,
$$

and this proves that $z$ is a fixed point. This completes the proof.

In what follows, we prove that Theorem 2.3 is still valid for $T$ being not necessarily continuous, assuming the following hypothesis in $X$ :

$$
\begin{aligned}
& \text { If }\left(x_{n}\right) \text { is a non-decreasing sequence in } X \text { such that } x_{n} \rightarrow x \\
& \text { then } x_{n} \leq x \text { for all } n \in N \text {. }
\end{aligned}
$$

Theorem 2.4 Let $(X, \leq)$ be a partially ordered set and suppose that there exists a metric d in $X$ such that $(X, d)$ is a complete metric space. Assume that $X$ satisfies (9). Let $T: X \rightarrow X$ be a non-decreasing mapping such that

$$
\psi(d(T x, T y)) \leq \phi(d(x, y)), \quad \forall x \geq y,
$$

where $\psi$ is a generalized altering distance functions and $\phi:[0, \infty) \rightarrow[0, \infty)$ is a right upper semi-continuous function with the condition $\psi(t)>\phi(t)$ for all $t>0$. If there exists $x_{0} \in X$ such that $x_{0} \leq T x_{0}$, then $T$ has a fixed point.

Proof Following the proof of Theorem 2.3 we only have to check that $T(z)=z$. As $\left(x_{n}\right)$ is a non-decreasing sequence in $X$ and $\lim _{n \rightarrow \infty} x_{n}=z$ the condition (9) gives us that $x_{n} \leq z$ for every $n \in N$ and consequently,

$$
\psi\left(d\left(x_{n+1}, T(z)\right)\right)=\psi\left(d\left(T\left(x_{n}\right), T(z)\right)\right) \leq \phi\left(d\left(x_{n}, z\right)\right) .
$$

Letting $n \rightarrow \infty$ and taking into account that $\psi$ is an altering distance function, we have

$$
\psi(d(z, T(z))) \leq \phi(0)
$$

Using condition of theorem we have $\phi(0)=0$, this implies $\Psi(d(z, T(z)))=0$. Thus, $d(z, T(z))=0$ or equivalently, $T(z)=z$.

Now, we present an example where it can be appreciated that the hypotheses in Theorems 2.3 and Theorems 2.4 do not guarantee uniqueness of the fixed point. An example appears in [12].

Let $X=\{(1,0),(0,1)\} \subset R^{2}$ and consider the usual order $(x, y) \leq(z, t) \Leftrightarrow x \leq z, y \leq t$. Thus, $(x, y)$ is a partially ordered set whose different elements are not comparable. Besides 
$\left(X, d_{2}\right)$ is a complete metric space considering $d_{2}$ the Euclidean distance. The identity map $T(x, y)=(x, y)$ is trivially continuous and non-decreasing and condition (1) of Theorem 2.4 is satisfied, since the elements in $X$ are only comparable to themselves. Moreover, $(1,0) \leq$ $T(1,0)=(1,0)$ and $T$ has two fixed points in $X$.

In what follows, we give a sufficient condition for the uniqueness of the point in Theorems 2.3 and 2.4. This condition is:

for $x, y \in X$ there exists a lower bound or an upper bound.

In [12] it is proved that condition (10) is equivalent to:

for $x, y \in X$ there exists $z \in X$ which is comparable to $x$ and $y$.

Theorem 2.5 Adding condition (11) to the hypotheses of Theorem 2.3 (resp. Theorem 2.4) we obtain the uniqueness of the fixed point of $T$.

Proof Suppose that there exist $z, y \in X$ which are fixed points. We distinguish two cases.

Case 1. If $y$ is comparable to $z$ then $T^{n}(y)=y$ is comparable to $T^{n}(z)=z$ for $n=0,1,2, \ldots$ and

$$
\begin{aligned}
\psi(d(z, y)) & =\psi\left(d\left(T^{n}(z), T^{n}(y)\right)\right) \\
& \leq \phi\left(d\left(T^{n-1}(z), T^{n-1}(y)\right)\right) \\
& \leq \phi(d(z, y)) .
\end{aligned}
$$

As we have the condition $\psi(t)>\phi(t)$ for $t>0$ we obtain $d(z, y)=0$ and this implies $z=y$.

Case 2. If $y$ is not comparable to $z$ then there exists $x \in X$ comparable to $y$ and $z$. Monotonicity of $T$ implies that $T^{n}(x)$ is comparable to $T^{n}(y)$ and to $T^{n}(z)=z$, for $n=0,1,2, \ldots$ Moreover,

$$
\begin{aligned}
\psi\left(d\left(z, T^{n}(x)\right)\right) & =\psi\left(d\left(T^{n}(z), T^{n}(x)\right)\right) \\
& \leq \phi\left(d\left(T^{n-1}(z), T^{n-1}(x)\right)\right) \\
& =\phi\left(d\left(z, T^{n-1}(x)\right)\right) .
\end{aligned}
$$

Hence, $\psi$ is a generalized altering distance function and we have the condition $\psi(t)>$ $\phi(t)$ for $t>0$, this gives us that $\left\{d\left(z, f^{n}(x)\right)\right\}$ is a non-negative decreasing sequence and, consequently, there exists $\gamma$ such that

$$
\lim _{n \rightarrow \infty} d\left(z, T^{n}(x)\right)=\gamma
$$

Letting $n \rightarrow \infty$ in (12) and, taking into account the properties of $\psi$ and $\phi$, we obtain

$$
\psi(\gamma) \leq \phi(\gamma)
$$

This and the condition $\psi(t)>\phi(t)$ for $t>0$ imply $\gamma=0$. Analogously, it can be proved that

$$
\lim _{n \rightarrow \infty} d\left(y, T^{n}(x)\right)=0
$$


Finally, as

$$
\lim _{n \rightarrow \infty} d\left(z, T^{n}(x)\right)=\lim _{n \rightarrow \infty} d\left(y, T^{n}(x)\right)=0
$$

the uniqueness of the limit gives us $y=z$. This finishes the proof.

Remark 2.6 Under the assumption of Theorem 2.3, it can be proved that for every $x \in X$, $\lim _{n \rightarrow \infty} T^{n}(x)=z$, where $z$ is the fixed point (i.e. the operator $f$ is Picard).

Remark 2.7 Theorem 1.2 is a particular case of Theorem 2.3 for $\psi$ being the identity function, and $\phi(t)=t-\psi(t)$. Theorem 1.3 is a particular case of our Theorem 2.3 for $\phi(t)$ being replaced by $\psi(t)-\phi(t)$. Theorem 1.4 is a particular case of Theorem 2.3 for $\psi$ being the identity function, and $\phi(t)=\beta(t) t$. Theorem 1.5 is also a particular case of Theorem 2.3 for $\psi$ and $\phi$ being continuous.

Example 2.8 The following are some generalized altering distance functions:

$$
\begin{aligned}
& \psi_{1}(t)= \begin{cases}0, & t=0, \\
{[t]+1,} & t>0,\end{cases} \\
& \psi_{2}(t)= \begin{cases}0, & t=0, \\
\lambda([t]+1), & t>0,\end{cases}
\end{aligned}
$$

where $\alpha>0$ is a constant.

$$
\psi_{3}(t)= \begin{cases}t, & 0 \leq t<1, \\ \alpha t^{2}, & t \geq 1,\end{cases}
$$

where $\alpha \geq 1$ is a constant.

We choose $\psi(t)=\psi_{3}(t)$ and

$$
\phi(t)= \begin{cases}t^{2}, & 0 \leq t<1, \\ \beta t, & t \geq 1,\end{cases}
$$

where $0<\beta<\alpha$ is a constant. By using Theorem 2.3, we can get the following result.

Theorem 2.9 Let $X$ be a partially ordered set and suppose that there exists a metric d in $x$ such that $(X, d)$ is a complete metric space. Let $T: X \rightarrow X$ be a continuous and nondecreasing mapping such that

$$
\begin{aligned}
& 0 \leq d(T x, T y)<1 \quad \Rightarrow \quad d(T x, T y) \leq(d(x, y))^{2}, \\
& d(T x, T y) \geq 1 \quad \Rightarrow \quad \alpha(d(T x, T y))^{2} \leq \beta d(x, y)
\end{aligned}
$$

for any $x, y \in X$. If there exists $x_{0} \in X$ such that $x_{0} \leq T x_{0}$, then $T$ has a fixed point. 


\section{Application to ordinary differential equations}

In this section we present two examples where our Theorems 2.3 and 2.4 can be applied. The first example is inspired by [17]. We study the existence of a solution for the following first-order periodic problem:

$$
\left\{\begin{array}{l}
u^{\prime}(t)=f(t, u(t)), \quad t \in[0, T], \\
u(0)=u(T),
\end{array}\right.
$$

where $T>0$ and $f: I \times R \rightarrow R$ is a continuous function. Previously, we considered the space $C(I)(I=[0, T])$ of continuous functions defined on $I$. Obviously, this space with the metric given by

$$
d(x, y)=\sup \{|x(t)-y(t)|: t \in I\}, \quad \text { for } x, y \in C(I),
$$

is a complete metric space. $C(I)$ can also be equipped with a partial order given by

$$
x, y \in C(I), \quad x \leq y \quad \Leftrightarrow \quad x(t) \leq y(t) m, \quad \text { for } t \in I .
$$

Clearly, $(C(I), \leq)$ satisfies condition (10), since for $x, y \in C(I)$ the functions $\max \{x, y\}$ and $\min \{x, y\}$ are least upper and greatest lower bounds of $x$ and $y$, respectively. Moreover, in [17] it is proved that $(C(I), \leq)$ with the above mentioned metric satisfies condition (9).

Now we give the following definition.

Definition 3.1 A lower solution for (13) is a function $\alpha \in C^{(1)}(I)$ such that

$$
\left\{\begin{array}{l}
\alpha^{\prime}(t) \leq f(t, \alpha(t)), \quad \text { for } t \in I, \\
\alpha(0) \leq \alpha(T)
\end{array}\right.
$$

Theorem 3.2 Consider problem (13) with $f: I \times R \rightarrow R$ continuous and suppose that there exist $\lambda, \alpha>0$ with

$$
\alpha \leq\left(\frac{2 \lambda\left(e^{\lambda T}-1\right)}{T\left(e^{\lambda T}+1\right)}\right)^{\frac{1}{2}}
$$

such that for $x, y \in R$ with $x \geq y$

$$
0 \leq f(t, x)+\lambda x-[f(t, y)+\lambda y] \leq \alpha \sqrt{g(x-y)}
$$

where $g(t):[0,+\infty) \rightarrow[0,+\infty)$ is a light upper semi-continuous function with $g(0)=0$, $g(t)<t^{2}, \forall t>0$. Then the existence of a lower solution for (13) provides the existence of an unique solution of (13).

Proof Problem (13) can be written as

$$
\left\{\begin{array}{l}
u^{\prime}(t)+\lambda u(t)=f(t, u(t))+\lambda u(t), \quad \text { for } t \in I=[0, T] \\
u(0)=u(T)
\end{array}\right.
$$


This problem is equivalent to the integral equation

$$
u(t)=\int_{0}^{T} G(t, s)[f(s, u(s))+\lambda u(s)] d s
$$

where $G(t, s)$ is the Green function given by

$$
G(t, s)= \begin{cases}\frac{e^{\lambda(T+s-t)}}{e^{\lambda T}-1}, & 0 \leq s<t \leq T, \\ \frac{e^{\lambda(s-t)}}{e^{\lambda T}-1}, & 0 \leq t<s \leq T .\end{cases}
$$

Define $F: C(I) \rightarrow C(I)$ by

$$
(F u)(t)=\int_{0}^{T} G(t, s)[f(s, u(s))+\lambda u(s)] d s .
$$

Note that if $u \in C(I)$ is a fixed point of $F$ then $u \in C^{1}(I)$ is a solution of (13). In what follows, we check that the hypotheses in Theorems 2.3 and 2.4 are satisfied. The mapping $F$ is non-decreasing, since we have $u \geq v$, and using our assumption. We can obtain

$$
f(t, u)+\lambda u \geq f(t, v)+\lambda v
$$

which implies, since $G(t, s)>0$, that for $t \in I$

$$
\begin{aligned}
(F u)(t) & =\int_{0}^{T} G(t, s)[f(s, u(s))+\lambda u(s)] d s \\
& \geq \int_{0}^{T} G(t, s)[f(s, v(s))+\lambda v(s)] d s=(F v)(t) .
\end{aligned}
$$

Besides, for $u \geq v$, we have

$$
\begin{aligned}
d(F u, F v) & =\sup _{t \in I}|(F u)(t)-(F v)(t)| \\
& =\sup _{t \in I}((F u)(t)-(F v)(t)) \\
& =\sup _{t \in I} \int_{0}^{T} G(t, s)[f(s, u(s))+\lambda u(s)-f(s, v(s))-\lambda v(s)] d s \\
& \leq \sup _{t \in I} \int_{0}^{T} G(t, s) \alpha \sqrt{g(u(s)-v(s))} d s .
\end{aligned}
$$

Using the Cauchy-Schwarz inequality in the last integral we get

$$
\begin{aligned}
& \int_{0}^{T} G(t, s) \alpha \sqrt{g(u(s)-v(s))} d s \\
& \quad \leq\left(\int_{0}^{T} G(t, s)^{2} d s\right)^{\frac{1}{2}}\left(\int_{0}^{T} \alpha^{2} g(u(s)-v(s)) d s\right)^{\frac{1}{2}} .
\end{aligned}
$$


The first integral gives us

$$
\begin{aligned}
\int_{0}^{T} G(t, s)^{2} d s & =\int_{0}^{t} G(t, s)^{2} d s+\int_{t}^{T} G(t, s)^{2} d s \\
& =\int_{0}^{t} \frac{e^{2 \lambda(T+s-t)}}{\left(e^{\lambda T}-1\right)^{2}} d s+\int_{t}^{T} \frac{e^{2 \lambda(s-t)}}{\left(e^{\lambda T}-1\right)^{2}} d s \\
& =\frac{1}{2 \lambda\left(e^{\lambda T}-1\right)^{2}} e^{(2 \lambda T-1)} \\
& =\frac{e^{\lambda T}+1}{2 \lambda\left(e^{\lambda T}-1\right)} .
\end{aligned}
$$

The second integral in (15) gives the following estimate:

$$
\begin{aligned}
\int_{0}^{T} \alpha^{2} g(u(s)-v(s)) d s & \leq \alpha^{2} g(\|u-v\|) \cdot T \\
& =\alpha^{2} g(d(u, v)) \cdot T .
\end{aligned}
$$

Taking into account (14)-(17) we have

$$
\begin{aligned}
d(F u, F v) & \leq \sup _{t \in I}\left(\frac{e^{\lambda T}+1}{2 \lambda\left(e^{\lambda T}-1\right)}\right)^{\frac{1}{2}} \cdot\left(\alpha^{2} g(d(u, v)) \cdot T\right)^{\frac{1}{2}} \\
& =\left(\frac{e^{\lambda T}+1}{2 \lambda\left(e^{\lambda T}-1\right)}\right)^{\frac{1}{2}} \cdot \alpha \cdot \sqrt{T} \cdot(g(d(u, v)))^{\frac{1}{2}}
\end{aligned}
$$

and from the last inequality we obtain

$$
d(F u, F v)^{2} \leq \frac{e^{\lambda T}+1}{2 \lambda\left(e^{\lambda T}-1\right)} \cdot \alpha^{2} \cdot T \cdot g(d(u, v))
$$

or, equivalently.

$$
2 \lambda\left(e^{\lambda T}-1\right) d(F u, F v)^{2} \leq\left(e^{\lambda T}+1\right) \cdot \alpha^{2} \cdot T \cdot g(d(u, v)) .
$$

By our assumption, as

$$
\alpha \leq\left(\frac{2 \lambda\left(e^{\lambda T}-1\right)}{T\left(e^{\lambda T}+1\right)}\right)^{\frac{1}{2}},
$$

the last inequality gives us

$$
2 \lambda\left(e^{\lambda T}-1\right) d(F u, F v)^{2} \leq 2 \lambda\left(e^{\lambda T}-1\right) \cdot g(d(u, v)),
$$

and, hence,

$$
d(F u, F v)^{2} \leq g(d(u, v)) .
$$


Put $\psi(t)=t^{2}$ and $\phi(t)=g(t)$. Obviously, $\psi$ is a generalized altering distance function, $\psi(t)$ and $\phi(t)$ satisfy the condition of $\psi(t)>\phi(t)$ for $t>0$. From (18), we obtain for $u \geq v$

$$
\psi(d(F u, F v)) \leq \phi(d(u, v)) .
$$

Finally, let $\alpha(t)$ be a lower solution for (13); we claim that $\alpha \leq F(\alpha)$. In fact

$$
\alpha^{\prime}(t)+\lambda \alpha(t) \leq f(t, \alpha(t))+\lambda \alpha(t), \quad \text { for } t \in I .
$$

We multiply by $e^{\lambda t}$,

$$
\left(\alpha(t) e^{\lambda t}\right)^{\prime} \leq[f(t, \alpha(t))+\lambda \alpha(t)] e^{\lambda t}, \quad \text { for } t \in I,
$$

and this gives us

$$
\alpha(t) e^{\lambda t} \leq \alpha(0)+\int_{0}^{t}[f(s, \alpha(s))+\lambda \alpha(s)] e^{\lambda s} d s, \quad \text { for } t \in I .
$$

As $\alpha(0) \leq \alpha(T)$, the last inequality gives us

$$
\alpha(0) e^{\lambda t} \leq \alpha(T) e^{\lambda T} \leq \alpha(0)+\int_{0}^{T}[f(s, \alpha(s))+\lambda \alpha(s)] e^{\lambda s} d s,
$$

and so

$$
\alpha(0) \leq \int_{0}^{T} \frac{e^{\lambda s}}{e^{\lambda T}-1}[f(s, \alpha(s))+\lambda \alpha(s)] d s .
$$

This and (19) give us

$$
\alpha(t) e^{\lambda t} \leq \int_{0}^{t} \frac{e^{\lambda(T+s)}}{e^{\lambda T}-1}[f(s, \alpha(s))+\lambda \alpha(s)] d s+\int_{t}^{T} \frac{e^{\lambda s}}{e^{\lambda T}-1}[f(s, \alpha(s))+\lambda \alpha(s)] d s
$$

and, consequently,

$$
\begin{aligned}
\alpha(t) & \leq \int_{0}^{t} \frac{e^{\lambda(T+s-t)}}{e^{\lambda T}-1} d s+\int_{0}^{t} \frac{e^{\lambda(s-t)}}{e^{\lambda T}-1}[f(s, \alpha(s))+\lambda \alpha(s)] d s \\
& =\int_{0}^{T} G(t, s)[f(s, \alpha(s))+\lambda \alpha(s)] d s \\
& =(F \alpha)(t), \quad \text { for } t \in I .
\end{aligned}
$$

Finally, Theorems 2.3 and 2.4 show that $F$ has an unique fixed point.

Example 3.3 In Theorem 3.2, we can choose the function $g(t)$ as follows:

(1) $g_{1}(t)=\ln \left(t^{2}+1\right)$;

(2)

$$
g_{2}(t)= \begin{cases}t^{3}, & 0 \leq t<1 \\ \frac{1}{2}, & t=1, \\ t, & 1<t<+\infty\end{cases}
$$


(3)

$$
g_{3}(t)= \begin{cases}t^{3}, & 0 \leq t \leq \frac{1}{2} \\ t-\frac{3}{8}, & \frac{1}{2}<t<+\infty\end{cases}
$$

The functions $g_{1}(t), g_{2}(t)$ are continuous and non-decreasing. The function $g_{3}(t)$ is right upper semi-continuous. If we choose $g(t)=g_{1}(t)$ in Theorem 3.2, we obtain the result of $[5]$.

Example 3.4 Consider the following first-order periodic problem:

$$
\left\{\begin{array}{l}
u^{\prime}(t)=\frac{\sin t}{e^{t}}-\beta x, \quad t \in[0, T] \\
u(0)=u(T)
\end{array}\right.
$$

Let

$$
f(t, x)=\frac{\sin t}{e^{t}}-\beta x, \quad x \in[0, \infty), t \in[0,1],
$$

then $f(t, x)$ is continuous. Further, for $x \geq y$, we have

$$
f(t, x)+\lambda x-[f(t, y)+\lambda y]=2(\lambda-\beta) \frac{x-y}{2} .
$$

We chose $\beta \in[0, \lambda]$ such that

$$
2(\lambda-\beta) \leq\left(\frac{2 \lambda\left(e^{\lambda T}-1\right)}{T\left(e^{\lambda T}+1\right)}\right)^{\frac{1}{2}}
$$

Taking $g(t)=\left(\frac{t}{2}\right)^{2}$ for all $t \in[0,+\infty)$, we have

$$
f(t, x)+\lambda x-[f(t, y)+\lambda y]=2(\lambda-\beta) \sqrt{g(x-y)} .
$$

By using Theorem 3.2, we know that the first-order periodic problem (20) has a unique solution.

A second example where our results can be applied is the following two-point boundary value problem of the second order differential equation:

$$
\left\{\begin{array}{l}
-\frac{d^{2} x}{d t^{2}}=f(t, x), \quad x \in[0, \infty), t \in[0,1], \\
x(0)=x(1)=0 .
\end{array}\right.
$$

It is well known that $x \in C^{2}[0,1]$ is a solution of $(20)$ that is equivalent to $x \in C[0,1]$ being a solution of the integral equation

$$
x(t)=\int_{0}^{1} G(t, s) f(s, x(s)) d s, \quad \text { for } t \in[0,1]
$$


where $G(t, s)$ is the Green function given by

$$
G(t, s)= \begin{cases}t(1-s), & 0 \leq t \leq s \leq 1 \\ s(1-t), & 0 \leq s \leq t \leq 1\end{cases}
$$

Theorem 3.5 Consider problem (21) with $f: I \times R \rightarrow[0, \infty)$ continuous and nondecreasing with respect to the second variable and suppose that there exists $0 \leq \alpha \leq 8$ such that for $x, y \in R$ with $x \geq y$

$$
f(t, x)-f(t, y) \leq \alpha \sqrt{g(x-y)},
$$

where $g(t):[0,+\infty) \rightarrow[0,+\infty)$ is a light upper semi-continuous function with $g(0)=0$, $g(t)<t^{2}, \forall t>0$. Then our problem (21) has a unique non-negative solution.

Proof Consider the cone

$$
P=\{x \in C[0,1]: x(t) \geq 0\} .
$$

Obviously, $(P, d)$ with $d(x, y)=\sup \{|x(t)-y(t)|: t \in[0,1]\}$ is a complete metric space. Consider the operator given by

$$
(T x)(t)=\int_{0}^{1} G(t, s) f(s, x(s)) d s, \quad \text { for } x \in P,
$$

where $G(t, s)$ is the Green function appearing in (22).

As $f$ is non-decreasing with respect to the second variable, for $x, y \in P$ with $y \geq x$ and $t \in[0,1]$, we have

$$
(T y)(t)=\int_{0}^{1} G(t, s) f(s, y(s)) d s \geq \int_{0}^{1} G(t, s) f(s, x(s)) d s \geq(T x)(t),
$$

and this proves that $T$ is a non-decreasing operator.

Besides, for $y \geq x$ and taking into account (23), we obtain

$$
\begin{aligned}
d(T y, T x) & =\sup _{t \in[0,1]}|(T x)(t)-(T y)(t)| \\
& =\sup _{t \in[0,1]}((T x)(t)-(T y)(t)) \\
& =\sup _{t \in[0,1]} \int_{0}^{1} G(t, s)(f(s, x(s))-f(s, y(s))) d s \\
& \leq \sup _{t \in[0,1]} \int_{0}^{1} G(t, s) \alpha \sqrt{g(x(s)-y(s))} \\
& \leq \sup _{t \in[0,1]} \int_{0}^{1} G(t, s) \alpha \sqrt{g(x(s)-y(s))} d s \\
& =\alpha \sqrt{\ln \left[\|y-x\|^{2}+1\right]} \sup _{t \in[0,1]} \int_{0}^{1} G(t, s) d s .
\end{aligned}
$$


It is easy to verify that

$$
\int_{0}^{1} G(t, s) d s=\frac{-t^{2}}{2}+\frac{t}{2}
$$

and that

$$
\sup _{t \in[0,1]} \int_{0}^{1} G(t, s) d s=\frac{1}{8}
$$

These facts, the inequality (24), and the hypothesis $0<\alpha \leq 8$ give us

$$
\begin{aligned}
d(T x, T y) & \leq \frac{\alpha}{8} \sqrt{g(x-y)} \\
& \leq \sqrt{g(\|x-y\|)}=\sqrt{g(d(x, y))} .
\end{aligned}
$$

Hence

$$
d(T y, T x)^{2} \leq g(d(x, y))
$$

Put $\psi(t)=t^{2}, \phi(t)=g(t)$, obviously $\psi$ is an altering distance function, $\psi$ and $\phi$ satisfy the condition of $\psi(t)>\phi(t)$, for $t>0$. From the last inequality, we have

$$
\psi(d(T x, T y)) \leq \phi(d(x, y))
$$

Finally, as $f$ and $G$ are non-negative functions

$$
T 0=\int_{0}^{1} G(t, s) f(s, 0) d s \geq 0
$$

and Theorems 2.3 and 2.4 tell us that $F$ has a unique non-negative solution.

Remark 3.6 In Theorem 3.5, we can choose $g(t)$ as $g_{1}(1), g_{2}(t)$, and $g_{3}(t)$ as well as in Theorem 3.2.

Example 3.7 Consider the following two-point boundary value problem of the second order differential equation:

$$
\left\{\begin{array}{l}
-\frac{d^{2} x}{d t^{2}}=\frac{\sin t}{e^{t}}+\frac{x}{1+\cos t \pi}, \quad x \in[0, \infty), t \in[0,1] \\
x(0)=x(1)=0
\end{array}\right.
$$

Let

$$
f(t, x)=\frac{\sin t}{e^{t}}+\frac{x}{1+\cos t \pi}, \quad x \in[0, \infty), t \in[0,1]
$$

then $f(t, x)$ is continuous and non-decreasing with respect to the second variable. Further, for $x \geq y$, we have

$$
f(t, x)-f(t, y)=\frac{x}{1+\cos t \pi}-\frac{y}{1+\cos t \pi} \leq \sqrt{\left(\frac{x-y}{2}\right)^{2}} .
$$


Taking $g(t)=\frac{t}{2}$ for all $t \in[0,+\infty)$. By using Theorem 3.2, we know that the two-point boundary value problem (25) has a unique non-negative solution.

\section{Competing interests}

The author declares that they have no competing interests.

Received: 29 May 2014 Accepted: 8 October 2014 Published: 3 November 2014

\section{References}

1. Khan, MS, Swaleh, M, Sessa, S: Fixed point theorems by altering distances between the points. Bull. Aust. Math. Soc 30(1), 1-9 (1984)

2. Dhutta, P, Choudhury, B: A generalization of contractions in partially ordered metric spaces. Appl. Anal. 87, 109-116 (2008)

3. Rhoades, BE: Some theorems on weakly contractive maps. Nonlinear Anal. 47, 2683-2693 (2001)

4. Aydi, H, Karapınar, E, Bessem, S: Fixed point theorems in ordered abstract spaces. Fixed Point Theory Appl. 2012 Article ID 76 (2012)

5. Nieto, JJ, Pouso, RL, Rodríguez-López, R: A fixed point theorem for contraction type maps in partially ordered metric spaces and application to ordinary differential equations. Proc. Am. Math. Soc. 135, 2505-2517 (2007)

6. Gordji, ME, Baghani, H, Kim, GH: Coupled fixed point theorems for contraction mapping induced by cone ball-metric in partially ordered spaces. Discrete Dyn. Nat. Soc. 2012, Article ID 981517 (2012)

7. Sintunavarat, W, Cho, YJ, Kumam, P: Existence, regularity and stability properties of periodic solutions of a capillarity equation in the presence of lower and upper solutions. Fixed Point Theory Appl. 2012, Article ID 128 (2012)

8. Obersnel, F, Omari, P, Rivetti, S: A generalization of Geraghty's theorem in partially ordered metric spaces and applications to ordinary differential equations. Nonlinear Anal., Real World Appl. 13, 2830-2852 (2012)

9. Sastry, K, Babu, G: Some fixed point theorems by altering distance between the points. Indian J. Pure Appl. Math. 30 641-647 (1999)

10. Amini-Harandi, A, Emami, H: A fixed point theorem for contraction type maps in partially ordered metric spaces and application to ordinary differential equations. Nonlinear Anal. 72, 2238-2242 (2010)

11. Harjani, J, Sadarangni, K: Fixed point theorems for weakly contraction mappings in partially ordered sets. Nonlinear Anal. 71, 3403-3410 (2009)

12. Burgić, D, Kalabušić, S, Kulenović, M: Global attractivity results for mixed monotone mappings in partially ordered complete metric spaces. Fixed Point Theory Appl. 2009, Article ID 762478 (2009)

13. Ćirić, L, Cakić, N, Rajović, M, Uma, J: Monotone generalized nonlinear contractions in partially ordered metric spaces. Fixed Point Theory Appl. 2008, Article ID 131294 (2008)

14. Gnana Bhaskar, T, Lakshmikantham, V: Fixed point theorems in partially ordered metric spaces and applications. Nonlinear Anal. 65, 1379-1393 (2006)

15. Lakshmikantham, V, Ćirić, L: Coupled fixed point theorems for nonlinear contractions in partially ordered metric spaces. Nonlinear Anal. 70, 4341-4349 (2009)

16. Nieto, JJ, Rodriguez-López, R: Contractive mapping theorems in partially ordered sets and applications to ordinary differential equations. Order 22, 223-239 (2005)

17. Nieto, JJ, Rodriguez-López, R: Existence and uniqueness of fixed point in partially ordered sets and applications to ordinary differential equations. Acta Math. Sin. 23, 2205-2212 (2007)

18. O'Regan, D, Petrusel, A: Fixed point theorems for generalized contractions in ordered metric spaces. J. Math. Anal. Appl. 341, 1241-1252 (2008)

19. Shatanawi, W, Postolache, M: Common fixed point theorems for dominating and weak annihilator mappings in ordered metric spaces. Fixed Point Theory Appl. 2013, Article ID 271 (2013)

20. Shatanawi, W, Postolache, M: Common fixed point results of mappings for nonlinear contraction of cyclic form in ordered metric spaces. Fixed Point Theory Appl. 2013, Article ID 60 (2013)

21. Karapınar, E, Shatanawi, W, Mustafa, Z: Quadruple fixed point theorems under nonlinear contractive conditions in partially ordered metric spaces. J. Appl. Math. 2012, Article ID 951912 (2012)

22. Shatanawi, W: Some fixed point results for a generalized-weak contraction mappings in orbitally metric spaces. Chaos Solitons Fractals 45, 520-526 (2012)

23. Harjani, J, Sadarangni, K: Generalized contractions in partially ordered metric spaces and applications to ordinary differential equations. Nonlinear Anal. 72, 1188-1197 (2010)

24. Yan, F, Su, Y, Feng, Q: A new contraction mapping principle in partially ordered metric spaces and applications to ordinary differential equations. Fixed Point Theory Appl. 2012, Article ID 152 (2012) 